\title{
An equivalence of multistatistics on permutations
}

\author{
Arthur Nunge $\|^{\| \dagger}$ \\ ${ }^{1}$ Laboratoire d'informatique Gaspard Monge, Université Paris-Est Marne-la-Vallée, 5 Boulevard Descartes, Champs- \\ sur-Marne, France
}

\begin{abstract}
We prove a conjecture of J.-C. Novelli, J.-Y. Thibon, and L. K. Williams (2010) about an equivalence of two triples of statistics on permutations. To prove this conjecture, we construct a bijection through different combinatorial objects, starting with a Catalan based object related to the PASEP.

Résumé. Nous prouvons une conjecture de J.-C. Novelli, J.-Y. Thibon et L. K. Williams de l'article (2010) à propos d'une équivalence de statistiques sur les permutations. Pour prouver cette conjecture, nous construisons une bijection passant par différents objets combinatoires en commençant par un objet de type Catalan relié au PASEP.
\end{abstract}

Keywords. Bijections, statistics, permutations, Catalan objects

\section{Introduction}

The algebra of noncommutative symmetric functions Sym [4] has been studied in algebraic combinatorics during the past twenty years. It contains many purely combinatorics problems as, for example, the explicit description of the relations between different bases through their transition matrices. In this paper we shall be interested in the basis introduced by Tevlin in [7], the so-called monomial basis of Sym, for which the transition matrices $\mathfrak{M}^{(n)}$ with the ribbon basis have been described in [5].

The authors proved that $\mathfrak{M}_{I, J}^{(n)}$ is equal to the number of permutations satisfying $\operatorname{GC}(\sigma)=I$ and $\operatorname{Rec}(\sigma)=J$ where GC and Rec are two statistics that will be recalled later. Some properties of this basis correspond to combinatorial properties of the PASEP (Partially ASymmetric Exclusion Process) which is a physical model in which particles hop back and forth (and in and out) of a one-dimensional lattice which is studied through the combinatorial object of permutation tableaux [2]. More precisely, in the study of the basis of Tevlin, the sum of the coefficients of the rows of the transition matrices corresponds to the number of permutation tableaux of given shapes.

In the PASEP context there exists a natural $q$-statistic on the permutation tableaux that becomes the number of 31-2 patterns on permutations (denoted by tot) thanks to a well-known bijection. This statistic allows us to define a $q$-analog of the basis of Tevlin as the functions whose transition matrices $\mathfrak{M}^{(n)}(q)$ with the ribbon basis are such that $\mathfrak{M}_{I, J}^{(n)}(q)$ is the sum of the $q^{\text {tot }(\sigma)}$ for all $\sigma$ satisfying $\operatorname{GC}(\sigma)=I$ and $\operatorname{Rec}(\sigma)=J$.

\footnotetext{
$\dagger$ Email: arthur.nunge@u-pem.fr.

1365-8050 @ 2016 Discrete Mathematics and Theoretical Computer Science (DMTCS), Nancy, France
} 
In [6], the authors studied those matrices in an algebraic way. However, the statistics GC and tot on permutations were not appropriate for an algebraic study. So the authors of [6] built suitable matrices $\widetilde{\mathfrak{M}}^{(n)}(q)$ for their algebraic purpose through other statistics on permutations also recalled later. $\widetilde{\mathfrak{M}}_{I, J}^{(n)}(q)$ is the sum of the $q^{\alpha(\sigma)}$ for all $\sigma$ satisfying $\operatorname{LC}(\sigma)=I$ and $\operatorname{Rec}(\sigma)=J$. They conjectured that their matrices are the same as the $\mathfrak{M}^{(n)}(q)$, or equivalently that the triples of statistics (GC, Rec, tot) and $(\mathrm{LC}, \mathrm{Rec}, \alpha)$ are equidistributed.

The aim of this paper is to prove this conjecture bijectively. As the triple of statistics (LC, Rec, $\alpha$ ) has a natural description on subexcedent functions, in order to gain in readability, we construct a bijection from permutations to subexcedent functions sending the triple of statistics (GC, Rec, tot) to (LC, DC, $\alpha$ ). The bijection is described as a succession of bijections through different combinatorial objects:

$$
\mathrm{P} \stackrel{\psi_{F V}}{\longleftrightarrow} \mathrm{WDP} \stackrel{\psi}{\longleftrightarrow} \mathrm{WDP} \stackrel{\phi_{2}}{\longleftrightarrow} \mathrm{DWSF} \stackrel{\phi_{1}}{\longleftrightarrow} \mathrm{SF}
$$

where P, WDP, DWSF, and SF are respectively Permutations, Weighted Dyck Paths, Decreasing Weighted Subexcedent Functions, and Subexcedent Functions which are all recalled or defined below. In Section 3 we shall describe the bijections $\psi_{F V}$ and $\psi$ which are natural bijections to obtain objects on which the statistics GC and tot are naturally defined. The object obtained is a pair consisting of a Catalan object and a weight. In Section 4 we begin by constructing a Catalan object associated with a weight from subexcedent functions through $\phi_{1}$ and then describe $\phi_{2}$, a Catalan bijection between decreasing subexcedent functions and Dyck paths. In Section 5 we prove the global result and give some properties associated with the global bijection.

\section{Notations and background}

\subsection{Permutations, compositions, and subexcedent functions}

Let us first fix our notations concerning permutations.

A recoil of a permutation $\sigma$ is a value $i$ such that $i+1$ is on the left of $i$. The recoil set of $\sigma$ is the set of the values of recoils. A 31-2 pattern of $\sigma$ is a pair $(i, j)$ such that $j>i+1$ and $\sigma_{i+1}<\sigma_{j}<\sigma_{i}$. We denote by $\operatorname{tot}(\sigma)$ the number of 31-2 patterns of $\sigma$. For example, tot $(528713649)=5$.

A composition of an integer $n$ is a sequence $I=\left(i_{1}, \ldots, i_{r}\right)$ of positive integers of sum $n$. In this case we write $I \mid=n$. The integer $r$ is called the length of the composition. The descent set of $I$ is $\operatorname{Des}(I)=\left\{i_{1}, i_{1}+i_{2}, \ldots, i_{1}+\cdots+i_{r-1}\right\}$. The recoil composition $\operatorname{Rec}(\sigma)$ of a permutation $\sigma \in \mathfrak{S}_{n}$ is the composition of $n$ whose descent set is the recoil set of $\sigma$.

The major index maj $(I)$ of a composition is the sum of the values in the descent set of $I$.

The Genocchi descent set [5] of a permutation $\sigma \in \mathfrak{S}_{n}$ is the set of values immediately followed by a smaller value (it is sometimes called descent top). The Genocchi composition of descents (or Gcomposition, for short) $\mathrm{GC}(\sigma)$ of a permutation is the integer composition $I$ of $n$ whose descent set is $\{d-1 \mid d \in \operatorname{GDes}(\sigma)\}$. For example, with $\sigma=528713649$, we have GDes $=\{5,6,7,8\}$ and $\mathrm{GC}(\sigma)=(4,1,1,1,2)$.

A subexcedent function of size $n$ is a word $u$ of size $n$ on the alphabet of nonnegative integers such that for each $i \in\{1, \ldots, n\}$ we have $u_{i} \leq n-i$. We denote by $\mathfrak{S F}_{n}$ the set of subexcedent functions of size $n$. They are enumerated by $n$ ! and in bijection with permutations through the Lehmer code. The statistic $L C$ is defined on a subexcedent function $u$ as follows. 
- Set $S=\emptyset$ and read $u$ from right to left. At each step, if the entry $k$ is strictly greater than the size of $S$, add the $(k-|S|)$-th element of the sequence $[1, n]$ not considering the elements of $S$.

- The set $S$ is the descent set of a composition $C$, and $\operatorname{LC}(\sigma)$ is the mirror image $\bar{C}$ of $C$.

This definition corresponds to the definition of LC in [6] after applying the Lehmer code on the inverse of the permutation.

For example, with $u=315503200, S$ is $\emptyset$ at first, then the set $\{2\}$ at the third step as the third letter from the right is a 2 and $S$ was empty, then $\{2,3\}$ (fourth step), then $\{2,3,5\}$ (sixth step), then $\{2,3,4,5\}$ (seventh step). Hence $C$ is $(2,1,1,1,4)$, so that $\mathrm{LC}(u)=(4,1,1,1,2)$.

From the same bijection between permutations and subexcedent functions, we define the number of inversions of a subexcedent function as the sum of its values. We also define the descent set of a subexcedent function as

$$
\operatorname{Des}(u)=\left\{i \in\{1, \ldots, n-1\} \mid u_{i}>u_{i+1}\right\}
$$

and the descent composition of $u$ (denoted by $\mathrm{DC}(u)$ ) as the composition of size $n$ whose descent set is $\operatorname{Des}(u)$. One can check that $\mathrm{DC}(315503200)=(1,3,2,1,2)$.

\subsection{The Françon-Viennot bijection}

The Françon-Viennot bijection, first described in [3], is a bijection between permutations and Laguerre histories. We shall describe a bijection between permutations and weighted Dyck paths (defined below) that can be obtained from the version of Corteel of the Françon-Viennot bijection described in [1] through a simple bijection between colored Motzkin paths and Dyck paths.

Definition 2.1 A weighted Dyck path of size $n$ is a Dyck path with $n$ up and $n$ down steps and a word $w$ of size $n$ called the weight. for all $i$, the weight satisfies $w_{i} \leq\left(h_{i}-1\right) / 2$ where $h_{i}$ is the height of the Dyck path between the $(2 i-1)$-th and $2 i$-th steps.

Figure 1 shows an example of a weighted Dyck path. We denote by $D_{i}$ the $i$-th step of the Dyck path. For a better readability we grouped the steps two by two between vertical dashed lines and only wrote strictly positive $w_{i}$ above steps $D_{2 i-1}$ and $D_{2 i}$. In this representation, $h_{i}$ is exactly the height of the point between steps $D_{2 i-1}$ and $D_{2 i}$.

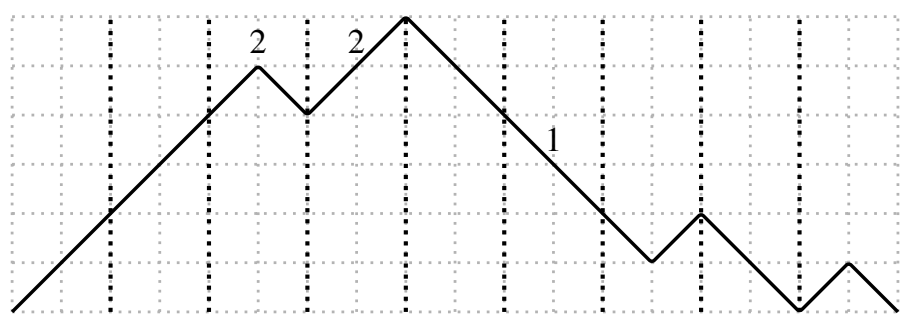

Fig. 1: A weighted Dyck path of size 9 .

It is difficult to directly show that the weighted Dyck paths are enumerated by $n$ ! but the FrançonViennot bijection $\psi_{F V}$ proves that it is the case for Laguerre histories so we obtain the result for weighted Dyck paths with the bijection described below. 
In the following algorithm, we compare each value of a permutation $\sigma$ with its two neighbors. We use the convention $\sigma_{0}=0$ and $\sigma_{n+1}=n+1$.

Algorithm 2.2 (Françon-Viennot) Let $\sigma \in \mathfrak{S}_{n}, j \in\{1, \ldots, n\}$ and $k=\sigma_{j}$, then in the Dyck path of $\psi_{F V}(\sigma)$ we have

- $D_{2 k-1}=D_{2 k}=/$ if $\sigma_{j}$ is a valley, i.e., $\sigma_{j-1}>\sigma_{j}<\sigma_{j+1}$,

- $D_{2 k-1}=D_{2 k}=\backslash$ if $\sigma_{j}$ is a peak, i.e., $\sigma_{j-1}<\sigma_{j}>\sigma_{j+1}$,

- $D_{2 k-1} D_{2 k}=\bigwedge$ if $\sigma_{j}$ is a double rise, i.e., $\sigma_{j-1}<\sigma_{j}<\sigma_{j+1}$,

- $D_{2 k-1} D_{2 k}=\bigvee$ if $\sigma_{j}$ is $a$ double descent, i.e., $\sigma_{j-1}>\sigma_{j}>\sigma_{j+1}$.

The weight is constructed as follows: $w_{k}$ is equal to the number of 31-2 patterns where $k$ represents the 2 .

For example, if we consider $\sigma=528713649$, its image by $\psi_{F V}$ is the weighted Dyck path of Figure 1 . One can check that the values 1,2 , and 4 are peaks for $\sigma$, the values 5, 6, and 8 are valleys, and so on. The 31-2 patterns are 52-3, 71-3, 52-4, 71-4, and 71-6 so that the weight is indeed $(0,0,2,2,0,1,0,0,0)$.

Let us also describe the reverse algorithm constructing a permutation from a weighted Dyck path $(D, w)$.

Algorithm 2.3 The permutation $\sigma$ is built iteratively by

- Initialization: $\sigma=0$;

- At the $k$-th step of the algorithm, replace the $\left(w_{k}+1\right)$-th $\circ$ of $\sigma$ by:

- $\circ$ o $\circ$ if $D_{2 k-1}=D_{2 k}=/$,

- $k \circ$ if $D_{2 k-1} D_{2 k}=\bigwedge$,

- ok if $D_{2 k-1} D_{2 k}=\bigvee$,

- $k$ if $D_{2 k-1}=D_{2 k}=\backslash$;

- The final permutation is obtained by removing the last $\circ$.

For the weighted Dyck path of Figure 1, the previous algorithm gives us:

$$
\begin{gathered}
\sigma=\circ \rightarrow \circ 1 \circ \rightarrow \circ 2 \circ 1 \circ \rightarrow \circ 2 \circ 13 \circ \rightarrow \circ 2 \circ 13 \circ 4 \circ \rightarrow 52 \circ 13 \circ 4 \circ \\
\rightarrow 52 \circ 13642 \circ \rightarrow 52 \circ 713642 \circ \rightarrow 528713642 \circ \rightarrow 528713649 \circ \rightarrow 528713649 .
\end{gathered}
$$

\section{Permutations to weighted Dyck paths}

Let us describe what happens to the three statistics on permutations through the Françon-Viennot bijection. 


\subsection{The statistics through the Françon-Viennot bijection}

Definition 3.1 Let $(D, w)$ be a weighted Dyck path of size $n$.

- The total weight of $(D, w)$ (noted $\operatorname{tw}(D, w))$ is the sum of the values of $w$.

- The descent set of $(D, w)$ is

$$
\operatorname{Des}(D, w)=\left\{i \mid w_{i}>w_{i+1}\right\} \cup\left\{i \mid w_{i}=w_{i+1}, D_{2 i}=/\right\}
$$

and the descent composition $\operatorname{DC}(D, w)$ is the composition of $n$ whose descent set is $\operatorname{Des}(D, w)$.

- The Genocchi descent set of $(D, w)$ is

$$
\operatorname{GDes}(D, w)=\left\{i \in[2, n] \mid D_{2 i-1}=\backslash\right\}
$$

then, as for permutations, the Genocchi composition of descent of $(D, w)$ (noted $\operatorname{GC}(D, w)$ ) is the composition of $n$ whose descent set is $\{d-1 \mid d \in \operatorname{GDes}(D, w)\}$.

On the weighted Dyck path $(D, w)$ of Figure 1 we have $\operatorname{tw}(D, w)=5$. The positions $i$ such that $D_{2 i-1}=\backslash$ are $\operatorname{GDes}(D, w)=\{5,6,7,8\}$ so that $\operatorname{GC}(D, w)=(4,1,1,1,2)$ and one can check that $\operatorname{DC}(D, w)=(1,3,2,1,2)$.

Lemma 3.2 Let $\sigma$ be a permutation of size $n$ and $(D, w)=\psi_{F V}(\sigma)$. We have the following properties:

- $\operatorname{tw}(D, w)=\operatorname{tot}(\sigma)$;

- $\operatorname{GC}(D, w)=\mathrm{GC}(\sigma)$;

- $\operatorname{DC}(D, w)=\operatorname{Rec}(\sigma)$.

Proof: The first two assertions come directly from our description of $\psi_{F V}$. To prove the last one, it is easier to work with $\psi_{F V}^{-1}$ constructing a permutation $\sigma$ from a weighted Dyck path $(D, w)$, see Algorithm 2.3. Let $k \in\{1, \ldots, n-1\}$. If $w_{k}<w_{k+1}$, then $k$ is to the left of $k+1$ in $\sigma$ so it is not a recoil for $\sigma$. Using the same idea, if $w_{k}<w_{k+1}$ then $k$ is a recoil for $\sigma$. Now, when $w_{k}=w_{k+1}$, we have a recoil in $k$ if and only if there is a new $\circ$ on the left of $k$ when we place it in $\sigma$ which happens if and only if $D_{2 k}=/$.

We now have a representation of the triple of statistics on the weighted Dyck paths but the definition of $\mathrm{GC}$ on those objects is not very natural because we need to consider the composition on a modified set. To simplify the second part of the construction, we transform the Dyck paths to obtain a more suitable statistic.

\subsection{An involution on Dyck paths}

Definition 3.3 Let $D$ be a Dyck path of size $2 n$. The Dyck path $\psi(D)$ is obtained by sending every pair of steps $\bigwedge$ or $\backslash /$ at positions $2 i, 2 i+1$ to one another.

We extend this definition to weighted Dyck paths by carrying the weight. Note that it is an involution. An example is given in Figure 2 where we apply $\psi$ to the weighted Dyck path of Figure 1.

Let us introduce the new statistics on weighted Dyck paths corresponding to DC and GC after applying $\psi$. 


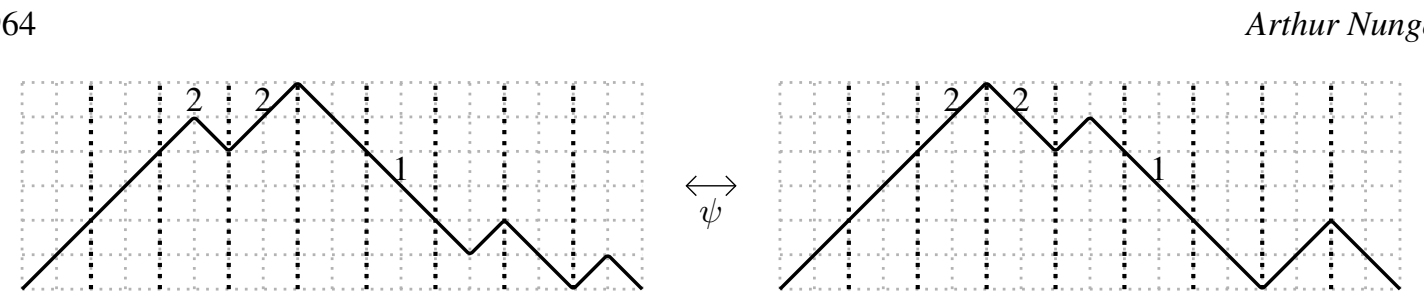

Fig. 2: An example of $\psi$.

Definition 3.4 Let us define $\mathrm{GC}^{0}$ and $\mathrm{DC}^{0}$ which are close to $\mathrm{GC}$ and $\mathrm{DC}$.

- The Genocchi descent set of type 0 of a weighted Dyck path of size $n$ is

$$
\operatorname{GDes}^{0}(D, w)=\left\{i \in[1, n-1] \mid D_{2 i}=\backslash\right\}
$$

and the Genocchi composition of descent of type 0 as the composition of $n$ whose descent set is $\operatorname{GDes}^{0}(D, w)$.

- The descent set of type 0 of $(D, w)$ is

$$
\operatorname{Des}^{0}(D, w)=\left\{i \mid w_{i}>w_{i+1}\right\} \cup\left\{i \mid w_{i}=w_{i+1}, D_{2 i+1}=/\right\}
$$

and the descent composition of type $0\left(\right.$ noted $\left.\mathrm{DC}^{0}(D, w)\right)$ is the composition of $n$ whose descent set is $\operatorname{Des}^{0}(D, w)$.

Lemma 3.5 Let $(D, w)$ be a weighted Dyck path, then

- $\operatorname{tw}(\psi(D, w))=\operatorname{tw}(D, w)$;

- $\mathrm{GC}^{0}(\psi(D, w))=\mathrm{GC}(D, w)$;

- $\operatorname{DC}^{0}(\psi(D, w))=\mathrm{DC}(D, w)$.

Proof: As $\psi$ does not change the weight of the Dyck path, the total weight is carried. For the other two statistics, one proves the claim by studying different cases depending on which steps are at positions $2 i$ and $2 i+1$.

\section{Subexcedent functions to weighted Dyck paths}

The aim of this section is to build a bijection from subexcedent functions to weighted Dyck paths sending the triple $(\mathrm{LC}, \mathrm{DC}$, inv $-\operatorname{maj}(\overline{\mathrm{LC}}))$ to $\left(\mathrm{GC}^{0}, \mathrm{DC}^{0}, \mathrm{tw}\right)$ where the statistic inv $-\operatorname{maj}(\overline{\mathrm{LC}})$ corresponds to the statistic $\alpha$ in the introduction. To do this, we construct a bijection from subexcedent functions to an intermediate object: decreasing weighted subexcedent functions which are represented by a Catalan object and a weight, and then construct a Catalan bijection between decreasing subexcedent functions and Dyck paths. 


\subsection{Subexcedent functions to decreasing weighted subexcedent functions}

Definition 4.1 Let us define a decreasing subexcedent function of size $n$ and $a$ weight for it.

- A subexcedent function $u$ of size $n$ is decreasing if the word obtained by removing all its zeroes is a strictly decreasing word.

- A weight of a decreasing subexcedent function is a word $w$ of size $n$ such that for all $k \in\{1, \ldots, n\}$, $w_{k}$ is smaller than or equal to the number of $i<k$ such that $0<u_{i}<n-k$ (i.e., the number of positive values on the left of $k$ that could be at position $k$ ).

For example, the subexcedent function $u=540300200$ is decreasing. For the associated weight, we can define a maximum weight as the weight for which each value is maximum. The maximum weight of $u$ is 012221000 so the weight 002201000 is acceptable whereas 000002000 is not.

We say that a value of a subexcedent function is weighted if the corresponding weight is nonzero.

Remark 4.2 The decreasing subexcedent functions are indeed Catalan objects since one can build a bijection with nondecreasing parking functions, the nondecreasing words whose $i$-th value is smaller than or equal to $i$ and whose first value is equal to one. First, reverse the subexcedent function, add one to all values and then replace the 1 values except the first one by their left neighboring value.

\subsubsection{Description of the bijection $\phi_{1}$ between subexcedent functions and de- creasing weighted subexcedent functions}

Our bijection $\phi_{1}$ can be described as an algorithm sorting a subexcedent function by moving successively the greatest value to its left.

Algorithm 4.3 Let $u$ be a subexcedent function of size $n$. Set weight $w$ to $0^{n}$.

- Step 1: define the pivot as the greatest value in u such that one of its occurrences has smaller or equal nonzero values to its left. If the pivot is not defined, the algorithm stops. Otherwise, let $k$ be the position of the rightmost occurrence of the pivot in $u$.

- Step 2: among the values smaller than or equal to the pivot on its left, let $i$ be the position of the rightmost occurrence of the greatest one. Modify the subexcedent function by decrementing $u_{i}$ by 1 and then swapping $u_{i}$ with $u_{k}$. Modify the weight by incrementing $w_{k}$. Go back to Step 1 .

Let us give an example with $u=315503200$. Our algorithm follows the steps:

- $u=315503200, P=000000000$, then pivot $=5, k=4$ and $i=3$;

- $u=315403200, P=000100000$, then pivot $=5, k=3$ and $i=1$;

- $u=512403200, P=001100000$, then pivot $=4, k=4$ and $i=3$;

- $u=514103200, P=001200000$, then pivot $=4, k=3$ and $i=2$;

- $u=540103200, P=002200000$, then pivot $=3, k=6$ and $i=4$;

- $u=540300200, P=002201000$ and the algorithm stops. 
At the end, $\phi_{1}(315503200)=(540300200,002201000)$.

Proposition 4.4 The map $\phi_{1}$ is a well-defined function from subexcedent functions to decreasing subexcedent functions.

Proof: It is clear that $\phi_{1}$ is well-defined for every subexcedent function $u$ and that the algorithm stops (for each pivot we have at most $n-1-$ pivot swaps).

The result is decreasing in the sense of Definition 4.1 because the algorithm sorts the subexcedent function.

To prove that the result is a decreasing weighted subexcedent function, we need to prove that the weight respects the constraints of Definition 4.1. Each value at position $j$ in the weight was increased at most once per pivot that ended on its left which makes even fewer values than the number of values on the left of $j$ at the end of the algorithm. Moreover, it was increased only if the pivot was at position $j$, which is possible only if the pivot is smaller than or equal to $n-j$. Finally $w$ respects the constraints of the definition.

Lemma 4.5 Consider Step 2 in Algorithm 4.3. After the exchange, we can find which values were swapped.

The position of the pivot is the rightmost position such that there exists a strictly smaller weighted value to its right, i.e., the rightmost position $i$ such that there exists $k>i$ with $w_{k}>0$ and $u_{i}>u_{k}$.

The pivot swapped with the nearest weighted value to its right whitch is always strictly smaller than the pivot.

Proof: We start by proving the second part of the lemma. We prove by induction on each different pivot that, with the notations of Algorithm 4.3, for all $i<j<k$, we have $w_{j}=0$. For the first pivot there is no problem as there is no weighted value to its left.

Assume the property is true when we change the value of the pivot. The weighted values are necessarily greater than or equal to the values on their left (up to the position where the previous pivot ended) so the new pivot must swap with all these weighted values. This ends the proof of this part of the lemma.

We also prove the other part of the lemma by induction. After the first exchange, the property is necessarily satisfied because of Step 2 of the algorithm. Assume that the property is true just after the exchange and for all the previous exchanges. There are two possibilities.

- If the pivot is not the same as in the previous Step 2, there is no weighted values to its right since it is the greatest value after the previous pivot and it begins at the starting position of the previous pivot or to its right. As there is no weighted values to its right, after its exchange, the pivot is the first greater value on the left of the rightmost weighted value.

- If the pivot stays the same, the property also holds since after the exchange, with the notations of Algorithm 4.3. $u_{k}$ is smaller than or equal to each weighted value to its right since the pivot swapped with all these weighted values. Moreover, each value between $u_{i}$ and $u_{k}$ is smaller than or equal to $u_{k}$.

Those cases prove that the property is true after each exchange.

The previous lemma shows that the map $\phi_{1}$ is injective by proving that we can find the previous pivot and the value with which value it is swapped at each step. We shall admit for now that decreasing weighted 
subexcedent functions are enumerated by $n$ ! (which will be proved in the next section) which proves the following proposition:

Proposition 4.6 The map $\phi_{1}$ is a bijection.

Note that the inverse map comes straightforward from Lemma 4.5

\subsubsection{The statistics through $\phi_{1}$}

Let us first define the new statistics on the decreasing weighted subexcedent functions.

Definition 4.7 Let $(u, w)$ be a subexcedent function of size $n$.

- The number of inversions $\operatorname{inv}(u, w)$ is the sum of the values of $u$ and $w$.

- The descent set of $(u, w)$ is

$$
\operatorname{Des}(u, w)=\left\{i \mid w_{i}>w_{i+1}\right\} \cup\left\{i \mid w_{i}=w_{i+1}, u_{i}>u_{i+1}\right\}
$$

and its descent composition $\operatorname{DC}(u, w)$ is the composition of $n$ whose descent set is Des $(u, w)$.

- The statistic $\mathrm{LC}$ on $(u, w)$ is the composition $\mathrm{LC}(u)$.

Remark 4.8 We shall make some remarks on the previous definitions.

1. The previous definitions are still correct if the weighted subexcedent function is not decreasing. Moreover, if we associate a null weight with a subexcedent function, those definitions give the same statistics than the usual ones on subexcedent functions.

2. Note that on a decreasing subexcedent function, the mirror composition of the statistic LC exactly corresponds to the composition whose descent set is the set of values of $u$. By defining the total weight statistic on the decreasing weighted subexcedent function as the sum of the values of its weight, we have directly $\operatorname{tw}(u, w)=\operatorname{inv}(u, w)-\operatorname{maj}(\overline{\operatorname{LC}(u, w)})$.

Lemma 4.9 Let $u$ be a subexcedent function, then:

- $\operatorname{tw}\left(\phi_{1}(u)\right)=\operatorname{inv}(u)-\operatorname{maj}(\overline{\operatorname{LC}(u)})$;

- $\operatorname{DC}\left(\phi_{1}(u)\right)=\mathrm{DC}(u)$;

- $\operatorname{LC}\left(\phi_{1}(u)\right)=\mathrm{LC}(u)$.

To prove the last point of this lemma we shall consider the exchanges of Algorithm 4.3 as a succession of elementary exchanges described by the following algorithm where $i$ and $k$ come from the notations of Algorithm 4.3 .

Algorithm 4.10 Set $j_{1}$ equal to $i$ and $j_{2}$ equal to $k$.

- Step 1: If $j_{1}=k-1$, go to step 2. Increment $u_{j_{1}+1}$ and then swap $u_{j_{1}}$ and $u_{j_{1}+1}$. Set $j_{1}=j_{1}+1$ and redo Step 1.

- Step 2: If $j_{2}=i$, the algorithm stops. Decrement $u_{j_{2}-1}$ and then swap $u_{j_{2}}$ and $u_{j_{2}-1}$. Set $j_{2}=j_{2}-1$ and redo Step 2 . 
For example, if at some point of Algorithm 4.3, we have $u=\ldots 4106 \ldots$ and we have to exchange 6 with 4, the exchanges of Algorithm 4.10 would be:

- we begin with Step 1:

$$
\begin{aligned}
& -u=\ldots 2406 \ldots ; \\
& -u=\ldots 2146 \ldots ;
\end{aligned}
$$

- and then apply Step 2:

$$
\begin{aligned}
-u & =\ldots 2163 \ldots ; \\
-u & =\ldots 2603 \ldots ; \\
-u & =\ldots 6103 \ldots
\end{aligned}
$$

Proof of Lemma 4.9: The proof of this lemma is based on the first point of Remark 4.8 and works by proving that the statistics inv, DC and LC do not change at each exchange of Algorithm 4.3

The inv statistic is not modified since each decrementation of a value of $u$ is balanced by a incrementation of a value of $w$.

In order to prove that the descent set of a weighted subexcedent function does not change when we are doing an exchange of Algorithm 4.3 , we have to study different cases depending on whether there is a descent at positions $i-1, i, k-1$, and $k$ or not. Let $\left(u^{(1)}, w^{(1)}\right)$ be the weighted subexcedent function before the exchange and $\left(u^{(2)}, w^{(2)}\right)$ the one after. For positions $k$ and $i-1$, one proves that $\left(u^{(1)}, w^{(1)}\right)$ has a descent in those positions if and only if it is the case for $\left(u^{(2)}, w^{(2)}\right)$. For the other positions one has to pay attention whether $i=k-1$ or not to prove the same property for both weighted subexcedent functions.

To prove that the statistic LC does not change at each step we prove that it is also true after each elementary exchange of Algorithm 4.10. Note that in both steps of the algorithm, the subexcedent functions before and after the exchange are two subexcedent functions $u^{(1)}$ and $u^{(2)}$ which differ only at positions $j$ and $j+1$. Moreover, if $u^{(1)}=\ldots a b \ldots$ with $a>b, u^{(2)}=\ldots b+1 a \ldots$, so we just have to prove that two such subexcedent functions satisfy $\operatorname{LC}\left(u^{(1)}\right)=\operatorname{LC}\left(u^{(2)}\right)$. To prove this property, one studies different cases depending on whether $a$ and $b$ add a value or not during the computation of $\operatorname{LC}\left(u^{(1)}\right)$.

\subsection{Decreasing weighted subexcedent functions to weighted Dyck paths}

Let us now describe the final bijection between decreasing weighted subexcedent functions and weighted Dyck paths. To describe it we construct a Dyck path from a decreasing subexcedent function and carry the weight. Then we show that it sends the triple of statistics of the decreasing weighted subexcedent functions to the triple of statistics of the weighted Dyck paths.

\subsubsection{Description of the bijection $\phi_{2}$ between decreasing weighted subexce- dent functions and weighted Dyck paths}

The map $\phi_{2}$ on a decreasing weighted subexcedent function of size $n$ is constructed by carrying the weight and constructing the Dyck path $D$ from the decreasing subexcedent function $u$ as follows. 
Algorithm 4.11 Set $D_{1}:=/$ and $D_{2 n}:=\backslash$. Then, for each $i$ in $\{1, \ldots, n-1\}$,

- set $D_{2 i}:=\backslash$ if $n-i$ is a value in $u$ and $D_{2 i}:=/$ otherwise;

- set $D_{2 i+1}:=\backslash$ if $u_{i}=0$ and $D_{2 i+1}:=/$ otherwise.

An example is given Figure 3 . The positions of the zeroes in the subexcedent function are $\{3,5,6,8,9\}$ which correspond to the positions $i$ such that $D_{2 i+1}=\backslash$. The values correspond to $n-i$ where $i$ are the positions where $D_{2 i}=\backslash$.

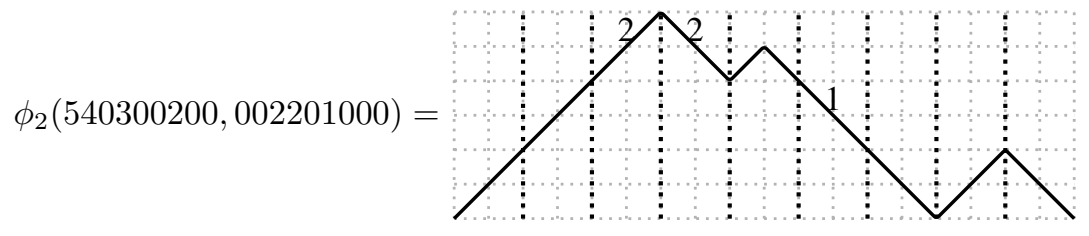

Fig. 3: An example of $\phi_{2}$.

Lemma 4.12 In the path $D$ constructed in Algorithm 4.11 let $h_{i}$ be the height between $D_{2 i-1}$ and $D_{2 i}$. Then, for all $i$, we have $\left(h_{i}-1\right) / 2$ equal to the number of values smaller than or equal to $n-i$ in $u$ to the left of $i$.

Proof: We prove this lemma by induction. For $i=1$, we have $\left(h_{1}-1\right) / 2=0$ and the property holds. Assume the property for a given $i$. To see that the property holds for $i+1$, one proves it by studying different cases depending on whether $u_{i+1}$ is equal to zero or not and $n-i-1$ is in $u$ or not.

Proposition 4.13 The map $\phi_{2}$ is well-defined from decreasing weighted subexcedent functions to weighted Dyck paths and is a bijection.

Proof: The image of a decreasing weighted subexcedent function is a path. Lemma 4.12 proves that the height of the path is always nonnegative and that $h_{n}=1$. As $D_{2 n}=\backslash$, the path is a Dyck path. Lemma 4.12 also gives us that the constraints on the weight of a decreasing weighted subexcedent function correspond to the constraints of its image.

As the constraints for the weights correspond exactly from one object to the other, we only need to prove that $\phi_{2}$ is a bijection from decreasing subexcedent functions to Dyck paths to prove that $\phi_{2}$ is a bijection on the weighted objects.

As decreasing subexcedent functions and Dyck paths are both enumerated by the Catalan numbers, we only need to prove that this map is injective. The only way to have the same image from two decreasing subexcedent functions is that the nonzero values and their positions are fixed, but as the subexcedent functions are decreasing, there is no choice in the order of those values.

Note that by proving that $\phi_{2}$ is a bijection we proved that decreasing weighted subexcedent functions are enumerated by $n$ ! so it finishes the proof of Proposition 4.6 .

\subsubsection{The statistics through $\phi_{2}$}

Lemma 4.14 Let $(u, w)$ be a decreasing weighted subexcedent function. We have

- $\operatorname{tw}(u, w)=\operatorname{tw}\left(\phi_{2}(u, w)\right)$; 
- $\mathrm{LC}(u, w)=\operatorname{GC}^{0}\left(\phi_{2}(u, w)\right)$;

- $\mathrm{DC}(u, w)=\mathrm{DC}\left(\phi_{2}(u, w)\right)$.

Proof: As the weight is carried, we have $\operatorname{tw}(u, w)=\operatorname{tw}\left(\phi_{2}(u, w)\right)$. For the descent set, as the weight is carried and the positions of the zeroes in $u$ correspond to the $\backslash$ steps at odd positions in $D$, we also have $\mathrm{DC}(u, w)=\mathrm{DC}\left(\phi_{2}(u, w)\right)$. Moreover, $\operatorname{LC}(u, w)$ is the mirror composition of the composition whose descent set is the nonzero values in $u$ and $\operatorname{GC}^{0}(D, w)$ is related to the $\backslash$ steps at even positions in $D$, so that we also have $\mathrm{LC}(u, w)=\mathrm{GC}^{0}\left(\phi_{2}(u, w)\right)$.

\section{Final result}

Theorem 5.1 Let I and J be two compositions of n, we have

$$
\sum_{\substack{u \in \mathfrak{S F}_{n} \\ \operatorname{DC}(u)=I \\ \mathrm{LC}(u)=J}} q^{\operatorname{inv}(u)-\operatorname{maj}(\bar{J})}=\sum_{\substack{\sigma \in \mathfrak{S}_{n} \\ \operatorname{Rec}(\sigma)=I \\ \mathrm{GC}(\sigma)=J}} q^{\operatorname{tot}(\sigma)} .
$$

Proof: To prove this theorem we apply successively the bijections defined in the previous sections to construct a permutation from a subexcedent function and we conclude by applying Lemmas 4.9, 4.14, 3.5. and 3.2 to prove the statistics correspondence.

Remark 5.2 This theorem proves Conjecture 6.2 of [6]. The other conjectures of [6] are directly obtained from this one.

Proposition 5.3 Define the left to right maximums of a permutation as the values with only smaller values to their left. In addition to carry the recoil composition of a permutation, the global bijection on permutations carries also the left to right maximums.

One proves this proposition by studying the statistic of left to right maximums through the bijections.

\section{References}

[1] S. CoRTEel, Crossings and alignments of permutations, Adv. in App. Math. 38(2) (2007), 149-163.

[2] S. CoRTEEL and L. WiLliams, Tableaux combinatorics for the asymmetric exclusion process, Adv. Appl. Math. 39 (2007), 293-310.

[3] J. FrançON and X. G. VIENnOT, Permutations selon leurs pics, creux, doubles montées et double descentes, nombres d'Euler et nombres de Genocchi, Disc. Math. 28(1) (1979), 21-35.

[4] I. M. Gelfand, D. Krob, A. Lascoux, B. Leclerc, V. S. Retakh, and J.-Y. Thibon, Noncommutative symmetric functions, Adv. in Math. 112 (1995), 218-348.

[5] F. Hivert, J.-C. Novelli, L. Tevlin and J.-Y. Thibon, Permutation statistics related to a class of noncommutative symmetric functions and generalizations of the Genocchi numbers, Sel. math., New ser. 15 (2009), 105-119.

[6] J.-C. Novelli, J.-Y. Thibon and L. Williams, Combinatorial Hopf Algebras, Noncommutative Hall-Littlewood Functions, And Permutation tableaux, Adv. Math. 224 (2010) 1311-1348.

[7] L. TeVlin, Noncommutative monomial symmetric functions, Proc. FPSAC'07, Tianjin, China. 